\title{
CORRESPONDENCE
}

\section{COVID-19-associated invasive pulmonary aspergillosis: high incidence or difficult diagnosis?}

\author{
Anahita Rouzé ${ }^{1,2}$, Elise Lemaitre ${ }^{1}$ and Saad Nseir ${ }^{1,2^{*}}$ (D)
}

(C) 2021 Springer-Verlag GmbH Germany, part of Springer Nature

\section{Dear Editor,}

We read with interest the letter by Prattes and colleagues [1], recently published in Intensive Care Medicine. The authors should be congratulated for their nice contribution. They report a high incidence (15.4\%) of coronavirus disease 2019 (COVID-19) associated invasive polmonary aspergillosis (CAPA), according to the 2020 ECMM/ISHAM consensus criteria [2], in a large multicenter cohort using a Fine and Gray competing risk model. Mortality in the intensive care unit (ICU) was also increased in patients with CAPA, as compared with those with no CAPA.

Although the incidence of CAPA is in line with some previous reports [3], other authors reported much lower incidence of CAPA in critically ill patients [4]. Important information is missing to correctly interpret the high incidence of CAPA reported by Prattes and colleagues. Firstly, how was the overall incidence calculated? The reported number of cases with possible, probable, and proven CAPA is 109 , which suggests that the incidence is even higher than that reported by the authors $(18.4 \%, 109$ of 592 patients). Secondly, were serum and bronchoalveolar lavage galactomannan performed routinely in the participating centers? As acknowledged by the authors, false positive results of galactomannan have been described and this might have artificially increased the incidence of CAPA. Thirdly, galactomannan was also performed in tracheal aspirate in 21 of 109 cases. To the best of our knowledge, the accuracy of tracheal aspirate galactomannan in diagnosing CAPA has not been evaluated.

\footnotetext{
*Correspondence: s-nseir@chru-lille.fr

'Médecine Intensive-Réanimation, CHU de Lille, F-59000 Lille, France

Full author information is available at the end of the article
}

Fourthly, were patients included consecutively? Based on the provided number of patients and centers, and study duration, it seems that only two COVID-19 patients were included per month per center, which is very low compared to the majority of ICUs during the pandemic. Fifthly, it would be interesting to provide the incidence of putative CAPA using Blot definition [5], as this definition was validated using histological data in a large multicenter cohort. Finally, the authors did not adjust for confounding factors regarding the incidence of CAPA and mortality, and no control group was included in this study. It would be interesting to know the incidence of invasive pulmonary aspergillosis in non-COVID patients in these centers.

\section{Author details \\ ${ }^{1}$ Médecine Intensive-Réanimation, CHU de Lille, F-59000 Lille, France. 2 INSERM U1285, CNRS, UMR 8576-UGSF-Unité de Glycobiologie Struc-} turale et Fonctionnelle, Université de Lille, F-59000 Lille, France.

\section{Declarations}

Conflicts of interest

SN: MSD, Gilead, Pfizer, Biomérieux, Mio Rad, Fischer and Paykel (lecture). Other authors: none.

\section{Publisher's Note}

Springer Nature remains neutral with regard to jurisdictional claims in published maps and institutional affiliations.

Accepted: 28 July 2021

Published online: 3 August 2021

References

1. Prattes J, Wauters J, Giacobbe DR et al (2021) Diagnosis and treatment of COVID-19 associated pulmonary apergillosis in critically ill patients:

\section{Springer}


results from a European confederation of medical mycology registry. Intensive Care Med. https://doi.org/10.1007/s00134-021-06471-6

2. Koehler P, Bassetti M, Chakrabarti A et al (2021) Defining and managing COVID-19-associated pulmonary aspergillosis: the 2020 ECMM/ISHAM consensus criteria for research and clinical guidance. Lancet Infect Dis 21:e149-e162. https://doi.org/10.1016/S1473-3099(20)30847-1

3. Bartoletti M, Pascale R, Cricca M et al (2020) Epidemiology of invasive pulmonary aspergillosis among COVID-19 intubated patients: a prospective study. Clin Infect Dis. https://doi.org/10.1093/cid/ciaa1065
4. Dellière S, Dudoignon E, Fodil S et al (2020) Risk factors associated with COVID-19-associated pulmonary aspergillosis in ICU patients: a French multicentric retrospective cohort. Clin Microbiol Infect. https://doi.org/ 10.1016/j.cmi.2020.12.005

5. Blot SI, Taccone FS, Van den Abeele A-M et al (2012) A clinical algorithm to diagnose invasive pulmonary aspergillosis in critically ill patients. Am J Respir Crit Care Med 186:56-64. https://doi.org/10.1164/rccm. 201111-19780C 